\title{
A Delay Arming Technical Scheme for Underwater Rocket Fuze Based on External Conformal Turbine
}

\author{
Shen Dezhang \\ Ministerial Key Laboratory of ZNDY \\ NUST, Nanjing, china
}

\author{
Zhang $\mathrm{He}$ \\ Ministerial Key Laboratory of ZNDY \\ NUST, Nanjing, china
}

\author{
Li Haojie \\ Ministerial Key Laboratory of ZNDY \\ NUST, Nanjing, china
}

\begin{abstract}
To solve underwater rocket fuze precise delay arming problem, a new delay arming technical scheme based on external conformal turbine is proposed, at the consider of underwater rocket fuze appearance and the particularity of underwater environment. The general scheme is presented, and designed the distance detection device, arming control circuit and actuator. A simulation test is developed to check the analysis and design in the end. The test results show that the scheme is feasible and effective, which can meet the needs of the underwater rocket fuze delay arming precision.
\end{abstract}

Keywords-External conformal turbine, Underwater rockets, Fuze, Delay arming

\section{INTRODUCTION}

Fuze safety design standards provides that fuze must have redundancy safety $\&$ arming functions, namely arming must rely on at least two different environment excitation in launch process [1]. To meet the aforementioned requirements, sensing environment information in the process of launching is the premise of a design of high performance fuze. The setback and centrifugal force in the process of launch are generally used as fuze S\&A system arming environment excitation. But these two environment excitation are hard to use in underwater rocket fuze because of its low setback and no rotation character.

Water is not compressible, so the shock wave of same strength in water damage about 4 times more than that in air $[2,3]$. Underwater rocket fuze must have the function of delay arming. The fuze traditional delay arming method mainly includes: gunpowder delay, fusible alloy, clockwork, quasi-fluid delay, air damped mechanism, ball rotor, safety belts and later all kinds of electromechanical delay arming device [4], mostly achieve delay arming through controlling time. The exterior ballistics parameters of underwater rocket is affected by water depth, to make the arming distance have low dispersion, delay arming device must be adaptive to projectile velocity.

Reference [5] and [6] put forward a technical solution of underwater rocket fuze delay arming device based on detecting the pressure differential of projectile body surface, and proposed a pressure detection point optimization method. This scheme is also vulnerable to angle of attack and water depth in the certain degree. A delay arming technical scheme for underwater rocket fuze based on external conformal turbine is proposed at consider of underwater rocket fuze appearance and the particularity of underwater environment. the preliminary experiment results show that he scheme is feasible.

\section{THE OVERALL SCHEME AND WORKING PRINCIPLE}

The underwater rocket fuze S\&A system structure diagram shows as shown in figure 1 , which achieve delay arming through the external conformal turbine.

Its working principle is: when the projectile moves underwater, turbine rotates driving by water flow, and there are certain function relation between the rotating speed and projectile velocity. Revolution counter detect the turbine real-time rotational speed and input it to MCU, MCU calculated projectile flight distance information, compares and makes the decision, outputs the arming control signal timely, Achieve arming action through executive circuit and actuating element, finish delay arming function. Because the turbine rotational speed is related to projectile velocity, this delay arming system is adaptive to bullet flying speed, theoretically can control arming distance within low dispersion.

\section{DESINE IMPLEMENTATION}

\section{A. Detection-mechanism design}

At present in the onshore conventional ammunition fuze, turbine alternator is widely used, mostly is built-in structure. From the inlet structure can be classified as straight inlet and side inlet two kinds. There has been some fuze extract the related features information from turbine alternator as arming control signal. To reduce the resistance, underwater rocket fuze is very thin, considering dimensional limitation and sealing requirements in water, this paper using external turbine realizing delay arming system distance signal detection. At the same time to reduce the influence on the bullet ballistic by external turbine, making the turbine appearance and the body keeps consistent, here call it conformal turbine.

The measurement of turbine rotational speed from the measurement principle can be classified as frequency measuring method and cycle measuring method, the main measurement technological means: tachogenerators, photoelectric measuring method, magnetic resistance measurement method and hall measurement method, etc. In 
this paper the scheme inlaid magnetic material in turbine, through the hall device detect turbine rotational speed. Distance detection mechanism principle shows as shown in figure 2.

When projectile traveling at high speed in underwater, the turbine rotates driven by water flow, small magnets set in turbine rotating together with turbine, hall element get turbine speed through testing the magnetic field changes. The scheme is simple in structure, low cost, and sealed performance is superior, because the rotation part and electrical part are completely isolate. To ensure magnetic field detection reliable, parts such as connecter 5 should not use ferromagnetic materials. Since there are electrical parts, parts like sensor seat 8 should use non-magnetic and nonconductive material such as plastic.

If $\mathrm{M}_{\omega}>\mathrm{M}_{\mathrm{f}}$, turbine starts rotating, according to the rigid body fixed axis rotation principle, turbine rotation differential equation:

$$
J \frac{d \omega}{d t}=M_{\omega}-M_{f}
$$

Where $\mathrm{J}$ is the rotational inertia of the rotor system, turbine, and bearings; $\mathrm{M}_{\omega}$ is the rotation driving moment of the turbine blades impacted by water flow; $\mathrm{M}_{\mathrm{f}}$ is general rotating resistance torque, mainly including mechanical friction resistance torque and fluid viscous resistance torque.

The driving moment can be calculated according to aerofoil theory, Rubin, Thompson, Blows etc. has established the lift force $\mathrm{L}$ and resistance $\mathrm{D}$ calculation formula of blade micro-unit area cdr according to aerofoil theory[7]:

$$
\begin{aligned}
& L=\frac{1}{2} \rho c C_{L}\left(\frac{V_{z}}{\cos \beta_{\infty}}\right)^{2} d r \\
& D=\frac{1}{2} \rho c C_{D}\left(\frac{V_{z}}{\cos \beta_{\infty}}\right)^{2} d r
\end{aligned}
$$

Where $\rho$ is fluid density, $\mathrm{kg} / \mathrm{m} 3 ; \mathrm{C}_{\mathrm{L}}$ is blade lift coefficients; $C_{D}$ is blade resistance coefficient; $C$ is blade chord length; $\beta_{\infty}$ is the angle of infinity far away velocity $U$ $\infty$ and axis at the case of single blade; $V_{z}$ is fluid incident speed parallel axis, $\mathrm{m} / \mathrm{s}$.

Mechanical friction resistance torque can use Palmgren calculation formula, and the calculation formula of fluid viscous resistance torque can establish according to viscosity fluid mechanics theory, this paper do not give the expatiatory detail.

The small magnets used RbFeB magnets. Due to the certain gap in the actual situation between the hall element and magnets surface, for providing data for the follow-up hall device selection, using LakeShore 450 gaussmeter measured the practical magnetic induction of area nearby some cylindrical little size $\mathrm{RbFeB}$ magnets, as is shown in table 1. In table the distance is axial distance of measure points from the end of the magnets.

Hall sensor using Hall Effect to realize the transformation from magnetic to electrical signal, it has a high sensitivity, excellent linearity, high stability, small volume and high temperature resistant characteristics, which is very suitable for the measurement of turbine speed. In this article, selected the Melexis company dual polarity hall lacth switch US1881, maximum switching frequency 10 $\mathrm{KHz}$, typical trigger and release threshold \pm 50 Gauss.

\section{$B$. Arming executive circuit and actuating element}

Figure 3 executive circuit of delay arming device. After power supply enabled, storage capacitor C1 was charged through the resistance to R1, its electric voltage can make electrical pinremoval reliable act after period of time. At this time due to no control signal output, thyristor cut-off, torage capacitor can not discharge to make electrical pinremoval act. When thyristor control electrode received the control signal, thyristor conduct, capacitor $\mathrm{C} 1$ discharge to electrical pinremoval, it acts immediately and pulls out arming pin, complete delay arming action. In circuit, R1 is charging current limit resistance, R2 and R3 are set to improve thyristor anti-interference ability, R4 is discharge resistance of $\mathrm{C} 1$, ensure $\mathrm{C} 1$ usually does not store electricity.

The delay arming action through electrical pinremoval completed. The main performance parameters of a certain type of electrical pinremoval shows as shown in table 2 .

electrical pinremoval reliable action need the energy $\mathrm{W} 1$, and after the thyristor conduct, storage capacitance discharge to electrical pinremoval through thyristor Q1, the electrical pinremoval gained energy W2 calculates as the following expression[5]:

$$
\begin{gathered}
W_{1}=I_{B}^{2} R_{B} t_{B} \\
W_{2}=\left(V_{d d^{-}}-V_{H}\right)^{2} \frac{R_{B} C_{1}}{2\left(R_{B}+R_{T}\right)}\left[1-e^{\frac{-2 t_{B}}{\left(R_{B}+R_{T}\right) C_{1}}}\right]
\end{gathered}
$$

Where $I_{B}$ is electrical pinremoval action current $(A), R_{B}$ is electrical pinremoval internal resistance $(\Omega), t_{B}$ is electrical pinremoval action time (s), $\mathrm{V}_{\mathrm{dd}}$ is power supply voltage $(\mathrm{V}), \mathrm{V}_{\mathrm{H}}$ is the corresponding anode voltage of thyristor maintain current $(\mathrm{V}), \mathrm{R}_{\mathrm{T}}$ is thyristor dynamic impedance $(\Omega), C_{1}$ is the storage capacitance value of capacitance $(\mathrm{F})$. Above two formulas can guide the key parameters of circuits design.

\section{PROTOTYPE AND SIMULATION TEST}

According to the scheme, product and assembled principle prototype, physical prototype shows as shown in figure 4.

Because the underwater dynamic tests prepare cycle is long, the cost is high, and get the relevant test parameters are difficult because of the limit of measurement methods. According to the movement relativity principle, this paper carried out preliminary simulation test in water tunnel for above detection device. The turbine rotational speed is measured in different flow velocities, and verified the feasibility of this mechanism.

Due to the limit of the water tunnel scale, intercept the front part of the prototype about $70 \mathrm{~mm}$, and making fittings installed on the testing part of water tunnel, control the water flow velocity through frequency changer regulating 
the motor speed, real-time measurement of turbine rotational accomplished by lead wires from the tail support bar center hole.

The part of data of the test results shows as shown in figure 5, this scheme can reliably detect real-time turbine rotational speed, and turbine rotational speed $\mathrm{R}$ and flow velocity $\mathrm{V}$ are linear relation in the testing condition, preliminarily confirm this solution is feasible.

\section{SUMMARIES}

From underwater rocket fuze accurate delay arming needs, combined with the underwater rockets fuze appearance and the particularity of the underwater environment, a delay arming technical solutions of underwater rocket fuze is proposed based on the external conformal turbine. The scheme characteristics is adaptive to speed, can better meet the practical needs. The preliminary simulation experiment shows that the scheme is feasible. The scheme can be used to realize the rocket fuze delay arming, may also used for other similar needs of underwater conventional ammunition, has a broad practicability.

\section{REFERENCES}

[1] GJB373A-97 Fuse safety design standard [S].

[2] HUANG Jian-song, KE Wen-qi, LE Xiu-hong, et al. Safety evaluation and protection on ship personnel subjected to simulation noncontact underwater explosion[J]. Chinese Journal of Nautical Medicine and H-yperbaric Medicine,2005,12(3):133-135.

[3] ZHANG Shou-zhong. Explosion and shock waves dy-namics[M]. Beijing: Ordnance Industry Press, 1993.

[4] SHI Geng-chen, LI Hua. Fuze's MEMS Delay Arming Device[J]. Journal of Detection \& Control,2008,30(3):1-4

[5] ]HOU Chao. System analysis and key technology s-tudy for underwater rocket fuzes[D]. Nanjing: $\mathrm{N}$-anjing University of Science and Technoloy,2008.

[6] SHEN De-zhang, ZHANG He, LI Hao-jie. Pressure Detection Point Optimization for Delay Arming Device of Underwater Rockets based on Numerical Simulation[J]. Journal of Ballistics,2010,22(4):36-40

[7] SUN L J. Research on reducing turbine flowmeter's sensitivity to viscosity change [D]. Doctoral dissertation, Tianjin: Tianjin university, 2004. 20-28

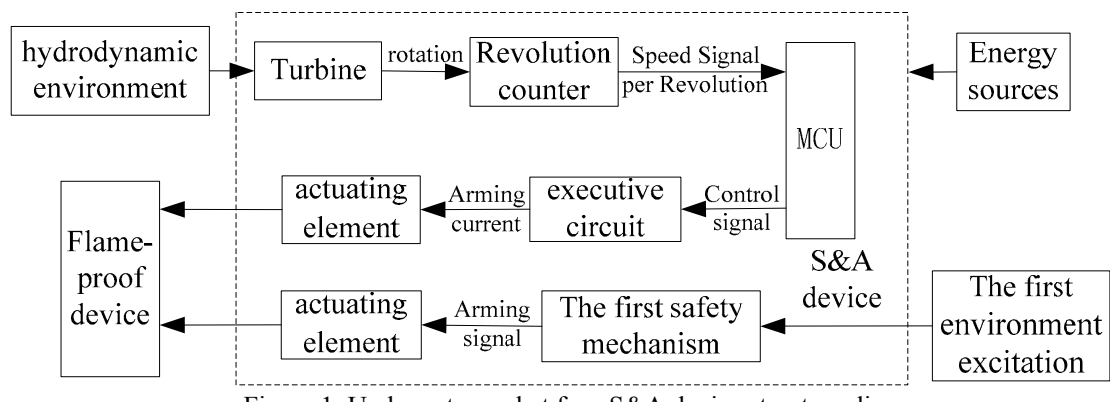

Figure 1. Underwater rocket fuze S\&A device structure diagram

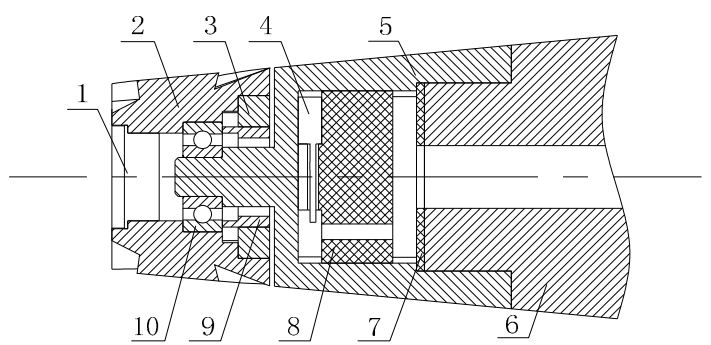

Figure 2. Detection-mechanism principle diagram

(1- end cover, 2- turbine, 3-magnet, 4-hall element, 5-connector, 6-fuze body, 7-seal ring, 8-sensor seat, 9-fastener, 10-bearing) 


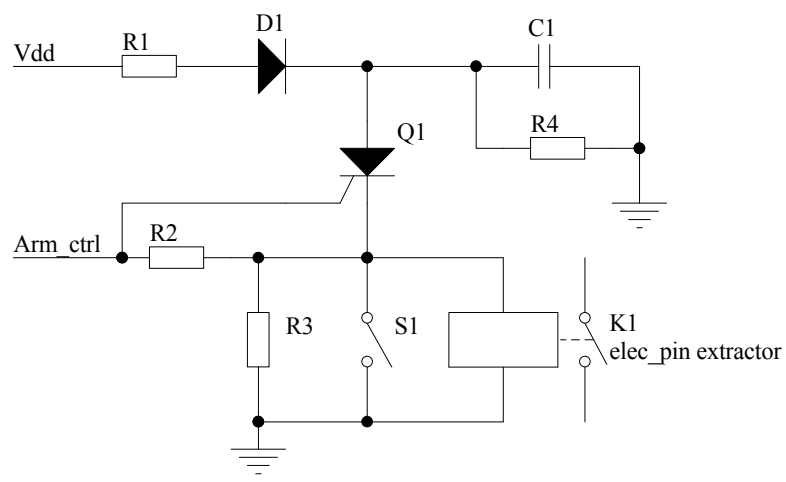

Figure 3. Arming executive control circuit

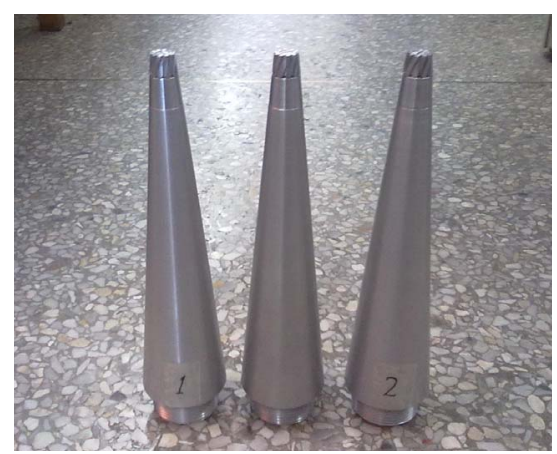

Figure 4. Physical prototype photogram Figure

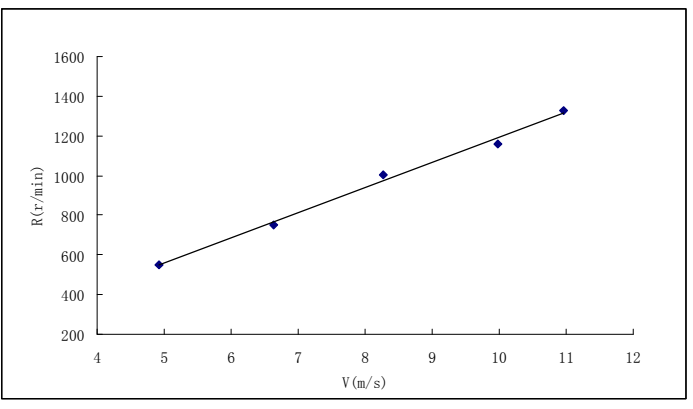

5. Test data curve

TABLE I. MAGNETIC INDUCTION NEARBY SOME RBFEB MAGNETS(GAUSS)

\begin{tabular}{cccc}
\hline Magnets size & $0 \mathrm{~mm}$ & $2 \mathrm{~mm}$ & $5 \mathrm{~mm}$ \\
\hline$\Phi 1 \times 2.5 \mathrm{~mm}$ & 1500 & 86 & 13 \\
$\Phi 1 \times 3 \mathrm{~mm}$ & 1800 & 95 & 14 \\
$\Phi 2 \times 1 \mathrm{~mm}$ & 2800 & 157 & 24 \\
$\Phi 2 \times 2 \mathrm{~mm}$ & 3000 & 200 & 41 \\
\hline
\end{tabular}

TABLE II. THE MAIN PERFORMANCE PARAMETERS OF A CERTAIN TYPE OF ELECTRICAL PINREMOVAL Introductions

Electric resistance

Antistatic performance

Misfire performance

Fire performance

Output performance

Action time

$5 \Omega+1.5 \Omega$

$500 \mathrm{PF}$, charge to $25000 \mathrm{~V}$ connect $5000 \Omega$ misfire

storage capacitor charge to $4 \mathrm{~V} \pm 0.4 \mathrm{~V}$, discharge to pinremoval, misfire

storage capacitor charge to $13 \mathrm{~V} \pm 0.5 \mathrm{~V}$, reliable fire

Drawing stroke not less than $1.4 \mathrm{~mm}$

Not more than $20 \mathrm{~ms}$ 